Proceedings of the International Conference "Quantum Optics IV", Jaszowiec, Poland, 1997

\title{
MEASUREMENT OF WAVE FIELDS
}

\author{
I.A. Walmsley, L. WaXer and C. Iaconis \\ Institute of Optics, University of Rochester, Rochester, NY 14627, USA
}

\begin{abstract}
Wave fields play a central role in both classical and quantum mechanics. Generally applicable methods for the characterization of (scalar) fields are outlined, and illustrated by experiment and simulation.
\end{abstract}

PACS numbers: 03.65.Bz, 42.50.Vk, 33.80.-b, 42.50.Dv

\section{Introduction}

Wave fields are the fundamental entities in both classical electrodynamics and quantum mechanics. The similarity of their governing dynamical equations suggests that similar strategies may be used to measure both classical and quantum fields. Indeed, numerous experiments in the recent past have demonstrated just this. But it is not this similarity alone that makes it important to consider their measurement within a single context, it is the very notion of manipulation of quantum systems (the idea of quantum state preparation or control) that demands it. Consider, for example the generation of a single photon "on demand", using the prescription, say, of Eberly and Law's "photon pistol" [1]. What does this mean, precisely? Nothing more than the specification of the quantum state of the field (a Fock state with eigenvalue unity) and a classical state of the field (the temporal mode in which the excitation is generated). Both pieces of information are necessary to appropriately specify what one means by the time of production of a photon. This problem resurfaces in all quantum measurement problems one needs to know both the basis in which one is making the measurement and the degree of freedom (or the mode) for which the measurement is defined.

To the extent that quantum state measurement is the same as state preparation, it might be argued that there is no need to consider state measurement procedures separately. Of course, in practice most measurement schemes end up being of the demolition variety - one infers from the detected signal what the state must have been at the detector before a signal was registered. This is quite a different situation than the ideal envisioned by von Neumann. But this aside, the situation may perhaps be likened to one of quality control, if one may adopt the industrial metaphor used by the state engineers. That is, the consumer needs to determine whether she has been sold the quantum system that meets her needs, in the state that she ordered. Thus she needs both a classical and a quantum field measurement apparatus. Of course in practice one must order (and measure) an ensemble of identically prepared states, and then use the apparatus to determine 
not only the quantum state and mode, but also the purity of the ensemble. Since presumably one would wish also to do an experiment with the purchased state, then the acquisition of two identical ensembles would, of course, be necessary, along with some "fair sampling" assumption consistent with good quality control.

\section{General strategies for wave field measurement}

\subsection{Quantum and classical measurements}

The quantities to be determined in the first instance, are, in the case of classical fields, the scalar optical field $E(x, t)$ (as a function of one of these variables only, at the moment) and in the case of quantum matter fields, the wave function $\Psi(x)$ (the dynamical dependence of this function being understood). In the case of the radiation field, the argument of the wave function is considered to be a field quadrature. In fact the measurements to be described can all be used to determine the purity of the state or the coherence of the field, in which case one seeks the density matrix (in position or momentum basis) or the two-point or two-time correlation function. We consider only fields or particles that are well localized, so that it is rather simple to define an ensemble for which the correlations may be evaluated. Thus the two-point correlation function for the electric field (taken to be time-stationary) can be defined as

$$
\Gamma\left(x, x^{\prime}\right)=\ll E^{*}(x) E\left(x^{\prime}\right) \gg,
$$

where the average is taken over a set of realizations of the experiment, repeated many times. This definition is analogous to the definition of the density matrix, which involves an average over all pure states that the system may potentially occupy. If one does not know a priori that the field is coherent or the state is pure, it is necessary to measure $\rho\left(x, x^{\prime}\right)$ or $\Gamma\left(x, x^{\prime}\right)$ and then to perform a test for coherence or purity. The test consists of assessing whether the function factorizes. That is, construct the parameter $\mu$, defined as

$$
0 \leq \mu=\frac{\int_{-\infty}^{+\infty} \mathrm{d} x \int_{-\infty}^{+\infty} \mathrm{d} x^{\prime}\left|\rho\left(x, x^{\prime}\right)\right|^{2}}{\left[\int_{-\infty}^{+\infty} \mathrm{d} x \rho(x, x)\right]} \leq 1 .
$$

Then, if $\mu=1$ the field is coherent, or the state is pure.

The similarity of the measurement problem in the case of (one-particle) quantum fields and the optical field arises not only because they obey similar dynamical equations, and can be represented by continuous complex functions of real variables, but also because they can be observed using only "square-law" type detectors*. A general measurement strategy for both can thus be worked out in the context of a strategy for either. We therefore consider the case of idealized linear quantum measurements. The analogous formulation for classical wave fields is related to the theory of optimal signal detection. There are two manipulations that one may perform on a quantum system; a unitary evolution $\widehat{U}_{\theta}$ where $\theta$ denotes a parameter, and a measurement $\widehat{\Pi}_{A}$, where $A$ denotes the outcome of the measurement. This is defined by

"For THz pulses it is possible to measure the dynamical ei actric field directly, so that this analogy does not hold. In the optical regime, however, the situation is quite different. 


$$
\widehat{\Pi}_{A}=\int \mathrm{d} A^{\prime} f\left(A \mid A^{\prime}\right)\left|A^{\prime}\right\rangle\left\langle A^{\prime}\right|
$$

where $f^{2}\left(A \mid A^{\prime}\right)$ is the conditional probability that the measurement will yield the outcome $A$ if the input was definitely in state $\left|A^{\prime}\right\rangle$.

According to Braginsky and Khalili [2] sequential measurements of two observables $\widehat{A}$ and $\widehat{A}^{\prime}$ are defined as linear if their commutator is a complex number. In the case of a sequence of unitary transformations (labeled by the set of parameters $\left\{\theta_{i}\right\}$ ) and measurements of the set of observables $\left\{\widehat{A}_{i}\right\}$, the probability that the result will be a set of real numbers $\left\{A_{i}\right\}$ is

$$
P\left(\left\{A_{i}\right\} ;\left\{\theta_{j}\right\}\right)=\operatorname{Tr}\left(\prod_{i, j} \widehat{\Pi}_{A_{i}} \widehat{U}_{\theta_{j}} \hat{\rho} \prod_{i, j} \widehat{\Pi}_{A_{i}}^{\dagger} \widehat{U}_{\theta_{j}}^{\dagger}\right) .
$$

For a single, ontinuous degree of freedom, the probability may be written as an overlap integral of the density matrix (taken here in the position representation) as

$$
P\left(\left\{A_{i}\right\} ;\left\{\theta_{j}\right\}\right)=\int_{-\infty}^{+\infty} \mathrm{d} x \int_{-\infty}^{+\infty} \mathrm{d} x^{\prime} \rho\left(x, x^{\prime}\right) F\left(x, x^{\prime} ;\left\{A_{i}\right\},\left\{\theta_{j}\right\}\right) .
$$

where the propensity function $F\left(x, x^{\prime} ;\left\{A_{i}\right\},\left\{\theta_{j}\right\}\right)$ depends on the meter and transformation parameters.

An entirely analogous expression may be derived for the photodetector signal at the output of a sequence of linear filters, at the input of which is an electric field specified by its correlation function $\left(\Gamma\left(x, x^{\prime}\right)\right.$ for the case of time-stationary fields and filters, or $\Gamma\left(t, t^{\prime}\right)$ for space-shift invariant fields and filters). In this case the analog of a meter (used for making a measurement) is an amplitude filter, and that of a unitary evolution is a phase-only filter. Then, denoting the filter transfer function parameters by $X_{i}$ and $\phi_{j}$ leads to

$$
S\left(\left\{X_{i}\right\} ;\left\{\phi_{j}\right\}\right)=\int_{-\infty}^{+\infty} \mathrm{d} x \int_{-\infty}^{+\infty} \mathrm{d} x^{\prime} \Gamma\left(x, x^{\prime}\right) F\left(x, x^{\prime} ;\left\{X_{i}\right\},\left\{\phi_{j}\right\}\right) .
$$

We may ask what are the minimal conditions for reconstructing this correlation function or density matrix? It is clear that any measurement apparatus must be capable of completely exploring the two-dimensional space of these functions even if one only wishes to measure a one-dimensional electric field or wave function. The simplest methods, therefore, require two linear operations. These take two forms, either in series, or in parallel. If one considers both meters and unitary-transformers two-port devices (the field enters at one port and exits in a modified form at the other) then the in-series arrangements consist only of two-ports. Any in-parallel arrangement must, though, include a way to divide the input field into parts, and must therefore contain at least one ancillary four-port device, such as a beam-splitter. The unused input port of such devices plays an important role in many quantum measurement apparati, but none at all in classical measurements.

In-series measurements consist of a meter or a unitary transformation, followed by a meter, as shown in Fig. 1a. In the case of two meters in sequence, it 
(a)

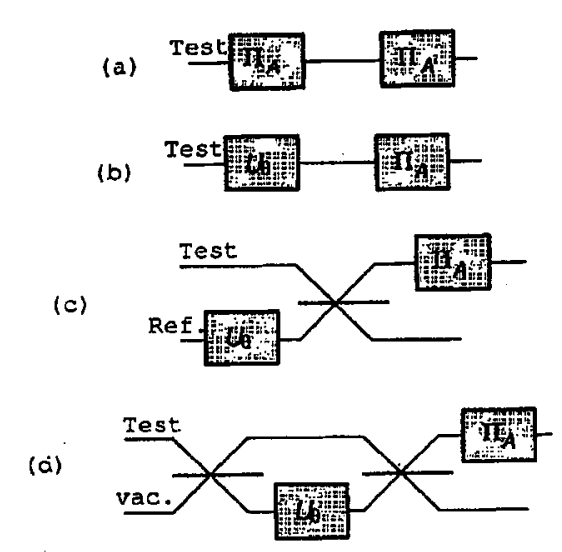

(b)

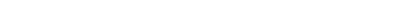

Fig. 1. The four minimal-operation in-series arrangements, and two in-parallel arrangements for field or state measurement for a single degree of freedom. Test denotes the unknown input field. $\widehat{\Pi}_{A}$ denotes a measurement and $\widehat{U}_{\theta}$ a unitary transformation of the field. (a) spectrographic (two-meter) (b) tomographic (unitary transform-meter) (c) test-plus reference. (d) self-referencing. In these figures "Ref" denotes a well-characterized reference field, and "vac" an empty input.

is straightforward to show that the meters must register complementary observables if the measured probability density is to be dependent on the phase of the density matrix. For the case of a unitary transformation and a meter, it is clear from Eq. (4) by cyclic permutation under the trace that the unitary operation must precede the meter, otherwise the probability density will be independent of the parameter characterizing the transformation.

The inversion method for these two schemes are quite different. The two-dimensional function returned by the two-meter method is a particular phase-space representation of the input state. If the first filter in the arrangement has a Gaussian conditional probability, and the second is precise (i.e. its conditional probability is a delta function) then the measured function is the $Q$-function, or its analog for a classical field. The inversion to the density matrix or correlation function is then via deconvolution. This class of measurement is termed spectrographic, since it involves characterizing the field by a spectrogram or a sonogram.

The method involving a unitary transform and a meter, shown in Fig. 1b, returns a set of one-dimensional positive functions (position or momentum distributions, for example) that depend also on the setting of the parameter(s) associated with the transformation. It is possible to invert this set of functions for any type of transformation [3], but it is particularly straightforward for transformations that are equivalent to dynamical evolution in a harmonic potential. In this case the transformation is characterized by a single parameter $\theta$ that ranges from $-\pi$ to $+\pi$. For a sufficiently large number of, say, position distributions, each with a different value of $\theta$ over this range, the discrete inverse Radon transform that is well known from computer-assisted tomography may be used to reconstruct the 
Wigner distribution of the field [4]. From this function it is straightforward to obtain the density matrix via a Fourier transform. This class of measurement is therefore labeled tomographic.

The second class of measurement involves two filters placed in parallel channels at the output ports of a four-port. Or two filters simultaneously in the two input channels of a second four port, at one of whose output ports is a meter. Schematic illustrations of these are shown in Figs. 1c and d. Both of these classes of measurement are essentially interferometric, and determine phase by comparison with a second field. The difference is that the first type of apparati use a known reference field for comparison, whereas the second type are self-referencing.

For classical fields, it is well known that the real part of the correlation function is simply related to the fringe pattern observed in two-beam interferograms. Even so, interferometry is not commonly used in measurements for coherence, and it has not yet been applied at all to the measurement of the state of quantum matter waves. On the other hand, the optical balanced homodyne detector may be thought of as an interferometer, so that the technique of homodyne tomography for characterizing quantum optical fields can be considered as either an in-series (if one takes the detector to measure the quadrature amplitudes directly) or in-parallel devices.

An important point in interferometric measurements is that there is always a second input field, whether or not it is explicitly manipulated by the experimenter. For example, in test-plus-reference type interferometers, Fig. 1c, the two input ports of the four-port are occupied by the test and reference fields. But in the self-referencing type (Fig. 1d) the second input port sees only the vacuum or no-particle state. The presence of this field may or may not compromise the precision of the measurement, but its presence must always be taken into account.

\subsection{Phase-space representations of in-series measurements}

Spectrographic and tomographic measurements can be clearly distinguished by their phase-space representations. Such spaces can be defined for both quantum and classical fields, and are based on the notion of conjugate variables. For example, the quantum oscillator phase-space variables are position and momentum, which are complementary in the sense that the commutator of the corresponding observables is a complex number, and the variance of the two observables taken in any state of the oscillator is greater than or equal to $\hbar$. The phase-space of a classical field can be thought of as also involving two classically conjugate variables, corresponding to two representations of the field. In the case of the optical field, these might be position and wave vector or time and frequency. In both cases the products of the variances of these quantities for any field is greater than or equal to $2 \pi$ according to Fourier's theorem - one may think of such Fourier pairs as "classically incompatible observables".

Thus for any complex field that is a function of a real variable, $\zeta(\chi)$, it is possible to define a Wigner representation of the correlation function of the field,

$$
W(\xi, \eta)=\int_{-\infty}^{+\infty} \mathrm{d} \chi \mathrm{e}^{\mathrm{i} \chi \eta} \Gamma\left(\xi-\frac{\chi}{2}, \xi+\frac{\chi}{2}\right) .
$$


For example, a temporally localized optical field may be represented by a.Wigner function of time and frequency in the chronocyclic phase space. This representation has all the analytic properties associated with the more familiar quantum Wigner function, including the property that it may be negative. Negativity in this context arises from the interference of classical waves rather than quantum waves, but nonetheless provides information about the coherence of the underlying field [5].

The output of spectrographic measurement apparati is a two-dimensional function of $\xi$ and $\eta$ (say, position and momentum, or time and frequency) that is related to the input field according to

$$
P(\xi, \eta)=\int_{-\infty}^{+\infty} \mathrm{d} \xi^{\prime} \int_{-\infty}^{+\infty} \mathrm{d} \eta^{\prime} W\left(\xi^{\prime}{ }^{\prime} \eta^{\prime}\right) H\left(\xi-\xi^{\prime}, \eta-\eta^{\prime}\right) .
$$

The function $H\left(\xi^{\prime}, \eta^{\prime}\right)$ is a phase-space "window" function through which one may gaze upon the input field. It is related to the propensity function defined by Eq. (5) by a transformation similar to Eq. (7). Since we assumed linear measurements (or filtering, in the case of classical fields) this function is a property of the apparatus alone. The window function occupies a minimum phase space area of $\hbar$ in the quantum case, or $2 \pi$ in the classical case, but only attains this minimum when the first measurement is imprecise and the second precise. It is easy to see why this should be so from a simple example, say the measurement of a short optical pulse. In the frequency-resolved optical gating (FROG) spectrographic method the first meter is a spectrometer or frequency meter, and the second a fast shutter, or time gate. It is obvious that a precise initial measurement of frequency will discard all information about the arrival time of the field, and thus render the shutter useless. Thus intuitively one might expect that the optimum situation is arrived at if the spectral filter bandwidth is comparable to the pulse spectral width and the shutter opening is very rapid. This turns out to be the case ${ }^{\dagger}$.

A cartoon of a spectrographic window function is shown in Fig. 2a. It is a well-localized entity in both transform variables, and moves about the phase
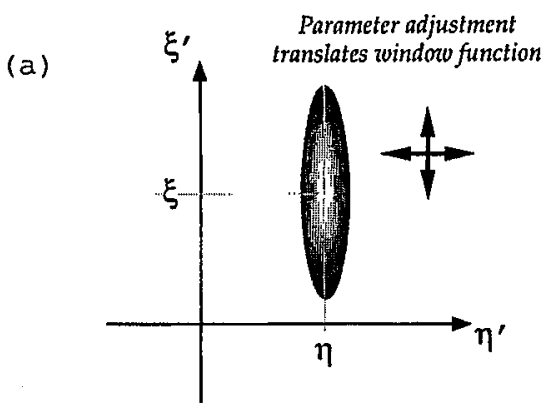

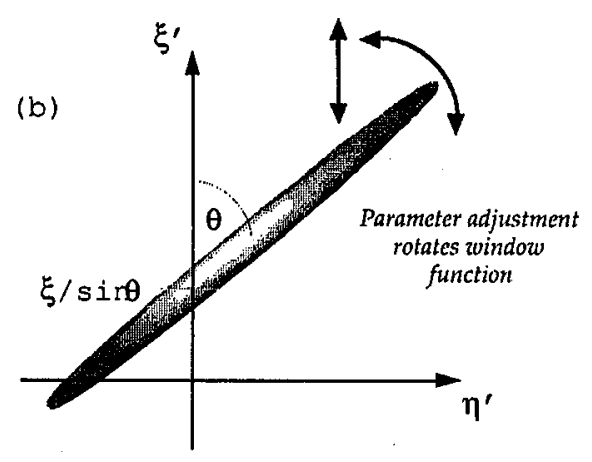

Fig. 2. Phase-space representations of window functions for in-series arrangements, (a) spectrographic. (b) tomographic.

†The formalism behind noninterferometric pulse characterization routines as well as an extensive list of references is given in Ref. [6]. 
space as the output variables $\xi$ and $\eta$ change, corresponding, in our example, to varying the time of opening of the shutter, and the tuning of the center of the spectrometer passband. These devices act as meters for the classical field in the sense that if one detects radiation having passed through the apparatus with a particular setting of these parameters, then one infers that the radiation contained that particular frequency during that particular time slot. The configuration space version of this situation has been discussed in connection with the measurement of spatially localized quantum wave functions by Raymer [7].

The output of tomographic measurement apparati are a set of one-dimensional functions of $\xi$ that are parametrized by the transformation variable $\theta$. In the case of harmonic tomography, these are related to the input field via

$$
P(\xi ; \theta)=\int_{-\infty}^{+\infty} \mathrm{d} \xi^{\prime} \int_{-\infty}^{+\infty} \mathrm{d} \eta^{\prime} W\left(\xi^{\prime}, \eta^{\prime}\right) T\left(\xi^{\prime}, \eta^{\prime} ; \xi, \theta\right)
$$

The function $T\left(\xi^{\prime}, \eta^{\prime} ; \xi, \theta\right)$ is also a phase-space window function, but has quite a different form than the spectrographic window function, as is shown in Fig. 2b. It rotates in the phase space as $\theta$ varies, and moves horizontally as $\xi$ varies. Again it is possible to show that the function occupies a minimum area of phase space for precise measurements of $\xi$, in which case the window function becomes a delta function, and $\theta$ is the angle between $T\left(\xi^{\prime}, \eta^{\prime} ; \xi, \theta\right)=\delta\left(\xi^{\prime} \sin \theta-\eta^{\prime} \cos \theta-\xi\right)$ and the $\xi$ axis. Then the measured probability distributions are projections of the Wigner function representing the field onto a set of rotated $\xi-\eta$ axes. The principles of tomography for quantum systems based on these ideas has been reviewed extensively by several authors $[8,9]$.

\subsection{Coherence-space representations of in-parallel measurements}

Interferometric measurements are more easily visualized in the space in which the correlation function resides. We shall label this the "coherence space" of the field. This might be a two-dimensional configuration space, in the case of $\Gamma\left(x, x^{\prime}\right)$ for example, or the two-dimensional momentum or wave vector space that is occupied by its conjugate function $\tilde{\tilde{\Gamma}}\left(k, k^{\prime}\right)$. These are two representations of the same underlying entity, of course, analogous to the position and momentum representations of the density matrix of a quantum particle. The relationship between these two representations and the phase-space densities discussed in Sec. 2.2 follows straightforwardly from Eq. (7). The Wigner phase-space density is obtained by a Fourier transform of the density matrix or correlation function in the $\xi$ representation, with respect to the difference of its two arguments. A second Fourier transform, this time with respect to the average of its two arguments, yields the $\eta$-space representation of the density matrix or correlation function. This may also be obtained by a second route in which the order of the Fourier transforms are inverted; the intermediate function in this case is the characteristic function of the Wigner distribution, which is known as the ambiguity function for classical fields.

There are two versions of the test-plus-reference interferometric measurement for quantum fields. In the first, a coherent-state field enters the reference port, after a phase shift characterized by the phase angle $\theta$. A precise $\xi$ measurement is made at the output ports. This leads to the method of optical homodyne tomography. 
In the second method, a vacuum enters the second input port, and precise $\xi$ and $\eta$ measurements are made simultaneously at the two output ports. Since these are incompatible observables, this arrangement corresponds to a spectrographic type of measurement, as discussed by Stenholm [10].

Self-referencing methods in which one of the input ports sees a vacuum are different. These correspond to looking at the region of coherence space occupied by the field using a point-like window representing a precise measurement of the $\eta$ or $\xi$ observable, a unitary transformation, parametrized by a displacement by amount $\delta \xi$ of one part of the field with respect to the other, and a phase-shift $\theta$. Because only a single observable is measured, there is no minimum area of phase space that the window function must occupy. The correlation function or density matrix can be constructed directly by a series of measurements of $\xi$ for various values of $\delta \xi$ and two values of $\theta$ separated by $\pi / 2$.

Given the direct access to the density matrix or correlation function that this class of measurement affords, one may question why it is not used more often when fields are to be characterized. The reason is that the unitary transformation and meter must be functions of the same observable, and it is rare that one is able to find both simple displacements and precise measurements in the same variable. For example, in the case of an ultrashort optical pulse, it is simple to displace the pulse in time - a delay line will do this - but impossible to measure the shape of the resulting temporal interference pattern, which contains beat frequencies as large as the optical frequency. On the other hand, it is easy to measure frequency precisely, but difficult to make a wavelength displacement of any significant magnitude.

\section{Spectrography and tomography}

\subsection{Emission tomography for quantum state reconstruction in matter}

The principles of tomography may be applied to the measurement of any material system in which the electronic and vibrational degrees of freedom are coupled. For systems in which the major mechanism for the damping of the electronic degree of freedom is radiation, tomographic reconstruction of the vibrational mode is accomplished from measurement of the spontaneous emission. An example of this is the reconstruction of the quantum state of the vibrational mode of a diatom in an excited electronic state from a measurement of its time-dependent fluorescence spectrum.

A molecule may be excited into nonclassical vibrational states, notably quadrature squeezed states [11] or classically distinguishable coherent superposition states $[12,13]$, by the application of a short optical pulse resonant with the electronic transition. Because the electronic and nuclear vibrational motions are coupled, a change in the electronic configuration causes a change in the nuclear configuration, so that when the molecule emits a photon and returns to the ground electronic state, as shown in Fig. 3, the wavelength of the emitted photon will depend on the location of the vibrational wave packet at the moment of emission. Thus the vibrational mode is allowed to evolve freely in a known potential for a certain time (this corresponds to the unitary evolution required for tomography) following which its position distribution is measured with a certain precision from the spectrum of spontaneous emission sampled at a given time [14]. 

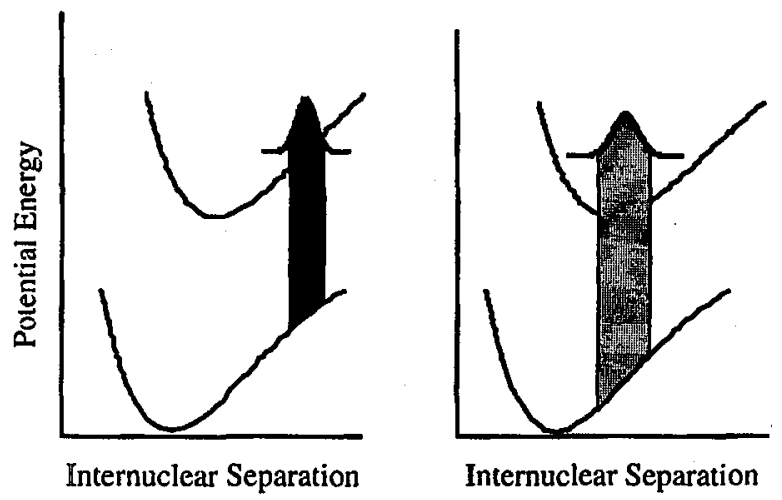

Fig. 3. Schematic illustration of the connection between quasi-instantaneous emission spectrum and wave packet position distribution for a molecular vibrational mode. A photon emitted by the molecule when the vibrational wave packet is at the outer turning point has a longer wavelength (black column) than one emitted when the packet is near the potential minimum (gray column).

These simple physical ideas may be put on a firmer footing using the notion of a time-dependent spectrum [15] based on an empirical scheme [16]. For example, in the case relevant to our experiments, the spontaneous radiation from a sample of molecules in the near-forward direction is sampled at some instant using a nonlinear-optical time gate, open for duration $\Gamma^{-1}$ near a time $T$ (which is referenced to the creation time of the wave packet by a preceding pump pulse). The spectrum of this temporal slice of fluorescence is then measured by a spectrometer with passband centered at frequency $\Omega$, and with spectral resolution $\gamma$ [16]. Thus the measured quantity is a two-parameter function $S(\Omega, T)$ : the time-dependent spectrum of spontaneous emission,

$$
S(\Omega, T)=K \sum_{n, m} \rho_{n m} \sum_{k} f_{k n} f_{k m}^{*} \exp \left(-\mathrm{i} \nu_{n m} T\right) g\left(\Omega-\omega_{21}^{n k}\right) g\left(\Omega-\omega_{21}^{m k}\right),
$$

where $\rho_{n m}$ is the vibrational density matrix in the excited electronic state $|2\rangle$ and $\nu_{n m}$ is the difference of vibrational frequencies in this state. $f_{k n}$ contains the details of the vibrational surfaces through the Franck-Condon factors. The unimportant constant $K$ specifies the correct units of the spectrum. The function $g$ is determined by the details of the time-gate and spectrometer response functions, and in our simple model is given by $g(\omega)=\exp \left[-\omega^{2} /\left(4 \Gamma^{2}\right)\right]$. It can be shown that the time-dependent spectrum of Eq. (10) corresponds to projections of a rotated phase-space density of the vibrational mode in the excited electronic state, with the position mapped to emitted wavelength in a way that depends on the details of the vibrational potentials ${ }^{\ddagger}$ [14]. The phase-space density is a smoothed version of the Wigner function, since there is an inherent uncertainty in the emission frequency when a time-gate is used to sample the spectrum. For the case of harmonic vibrational potentials, the density can be obtained from the spectrum using the in-

\footnotetext{
$\$$ The desired information can also be determined by photoelectron spectroscopy, see Ref. [17].
} 
verse Radon transform [14]. But in general molecular vibrations are not harmonic, so different inversion methods must be used to implement tomography.

A small, easily invertable linear system can be developed from Eq. (10). A time series is obtained from the time-dependent spectrum by sampling. The time series is a sampling of the truncated spectrum defined $S^{\prime}(\Omega, T)=S(\Omega, T) \times G(T ; \tau)$ where $G(T ; \tau)$ is a sampling window of length $\tau$. The Fourier transform of this series with respect to $T$ is

$$
\tilde{S}^{\prime}(\Omega, \nu)=K \sum_{n, m} \rho_{n m} \sum_{k} f_{k n} f_{k m}^{*} g\left(\Omega-\omega_{21}^{m k}\right) g\left(\Omega-\omega_{21}^{n k}\right) \widetilde{G}\left(\nu-\nu_{n m}\right) .
$$

For a particular value of $\nu$, chosen typically to lie near a maximum of $\widetilde{G}\left(\nu-\nu_{n m}\right)$, there will be several $\rho_{n m}$ which contribute to $\widetilde{S}^{\prime}(\Omega, \nu)$. It is possible to resolve the contribution of each density matrix element to the time-series spectrum at frequency $\nu$ by forming a linear system consisting of a set of different time series, each associated with different values of the frequency filter setting, $\Omega$. This system can then be inverted to find the particular contributing density matrix elements [18].

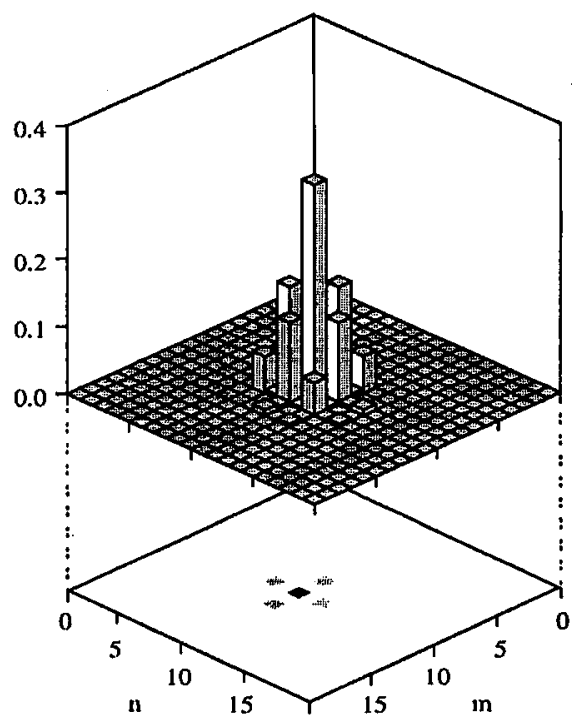

Fig. 4. Reconstruction of a classically distinguishable coherent superposition state for an anharmonic vibrational mode, from a simulated time-dependent emission spectrum, in the basis of eigenstates $|n\rangle,|m\rangle$ of the vibrational mode in the excited electronic state. The upper histogram represents the modulus of the density matrix elements and lower portion represents the errors between reconstructed and actual matrix elements.

To illustrate the efficacy of this method, Fig. 4 shows a simulated reconstruction of the reduced density matrix for a molecular vibration in the $A^{1} \Sigma_{\mathrm{u}}^{+}$ state of the sodium dimer, measured by its fluorescence to the $X^{1} \Sigma_{\mathrm{g}}^{+}$state. The method is quite robust, and is capable of reconstructing a complicated mesoscopic Schrödinger-cat state, simulated by superposing two quasi-coherent-state wave 
packets which are separated by one half a vibrational period. The mean vibrational quantum number of each wave packet was set at $\bar{n}=9$, and about 8 states were populated around this quantum number. The temporal resolution was taken to be $20 \%$ of the classical vibrational period $(310 \mathrm{fs})$, or $\Gamma=0.0167 \mathrm{fs}^{-1}$, and the total sampling time was $\tau=23 \mathrm{ps}$, corresponding to the one-quarter fractional revival period for this molecular state. In this case we used up to 13 different time scans (each corresponding to a particular value of $\Omega$ ). In order to ensure a stable inversion, the values of $\Omega$ were chosen to be equally spaced across the fluorescence spectrum of the molecular wave packets (i.e. in wavelength, between $630 \mathrm{~nm}$ and $810 \mathrm{~nm}$ corresponding to the wave packet classical turning points).

\subsection{Chronocyclic spectrography for classical ultrashort optical pulse shape reconstruction}

Tomography has also been proposed as a method for the measurement of the (classical) electric field of an ultrashort optical pulse ${ }^{\S}$. It has not yet been implemented experimentally, since, for technical reasons, it is much easier to make the measurements using a spectrographic method. The most widely studied of the spectrographic methods is FROG, but there are alternates, such as a version of Treacy's dynamic spectrogram known as temporal analysis of spectral components or TASC.

These two techniques measure two different types of spectrogram: based on either resolving the spectrum of temporally filtered components, as in FROG [20-23], or time-resolving each component of the pulse's spectrum as in TASC $[24,25]$ or other methods [26]. The two permutations of spectrometer and time-gate make up the two simplest experimental arrangements for spectrographic measurement of pulsed optical fields.

The dynamic spectrogram was shown by Chilla and Martinez [25] to possess a simple phase retrieval algorithm in the limit of precise frequency measurements. A better estimate of the input pulse field, using an iterative phase-retrieval algorithm, is available if the measurement is imprecise, however, and the full temporal dependence of each spectral component is measured.

Our experimental demonstration of TASC measured the output of a conventional Kerr-lens modelocked Ti:sapphire laser operating at $808.5 \mathrm{~nm}$, producing pulses $80-90 \mathrm{fs}$ in duration, with a spectral FWHM of $11.8 \mathrm{~nm}$. The pulses passed through an optical isolator before being measured. This type of source has also been measured using FROG [27].

The apparatus, shown schematically in Fig. 5a was a modified Michelson interferometer, in one arm of which was a quasi-zero-dispersion grating spectrometer, with adjustable passband center frequency $\omega_{\mathrm{c}}$. In the reference arm, the input pulse was reflected by a corner cube and a plane mirror. The corner cube was moveable to provide the variable temporal delay $\tau$. These two pulses are combined in a non-collinear geometry onto a $300-\mu \mathrm{m}$-thick nonlinear crystal, resulting in second-harmonic generation ( $\mathrm{SHG}$ ) which is detected by a photomultiplier tube (PMT). The nonlinear optical interaction of SHG forms the time gate for the

\footnotetext{
$\S$ For an introduction to pulse shape measurement methods see Ref. [19].
} 

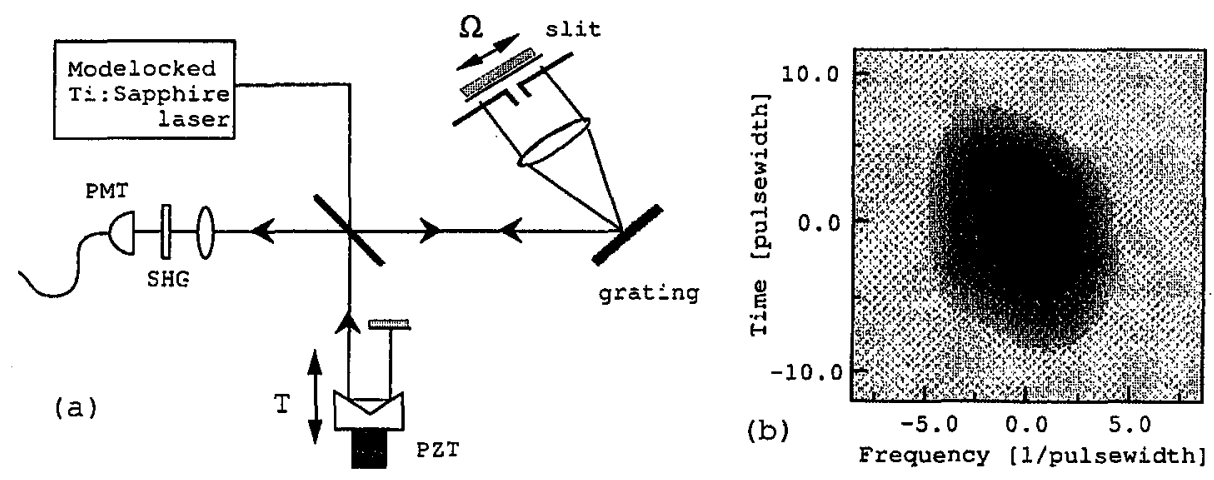

Fig. 5. (a) Apparatus for the characterization of an ultrashort optical pulse via TASC. (See text for description.) (b) Experimentally measured spectrogram measured using the apparatus of (a).

spectrally filtered pulse from the other arm. The spectrogram is obtained from the resulting cross-correlations, which are recorded as a function of $\Omega$ and $T$.

Figure $5 \mathrm{~b}$ shows the spectrogram recorded with spectrometer resolution of one-tenth the spectral bandwidth of the input pulse. The spectrogram displays a slight negative chirp, arising primarily from the residual chirp in the spectral filter combined with the pulse's phase structure due to the dispersion of the optical isolator. This intuitive spectrographic representation of the pulse is a feature that TASC shares with polarization-gate-FROG, which uses a third-order nonlinearity.

The extraction of the input spectral field $\widetilde{E}_{\text {in }}(\omega)$ from the TASC spectrogram involves a two-dimensional phase retrieval problem, which is well known to yield unique solutions [20]. We apply an iterative algorithm based on the method of generalized projection similar to that of FROG [28] to perform the two-dimensional phase retrieval [24].

The amplitude and phase of the pulse that are reconstructed from the sonogram exhibit the correct phase structure expected from the passage of a nominally 80 fs transform-limited pulse from the Ti:sapphire laser through the dispersive optical isolator.

\section{Interferometry}

\subsection{Self-referencing interferometry for characterizing the spatial coherence of classical optical fields}

It is well known that the real part of $\Gamma\left(x, x^{\prime}\right)$ is simply related to the fringe pattern observed in two-beam interferograms. Despite this, interferometric measurements have been sparingly applied to the measurement of fields with an arbitrary correlation function [29-34].

It is possible, however, to construct an interferometer capable of measuring the space-shift variant two-point correlation function for fields at a remote plane with arbitrary spatial coherence in a simple, accurate and efficient manner [35].

The apparatus consists of a Sagnac interferometer, shown in Fig. 6a, consisting of a polarization-insensitive nominally $50 / 50$ beamsplitter and two mirrors. 


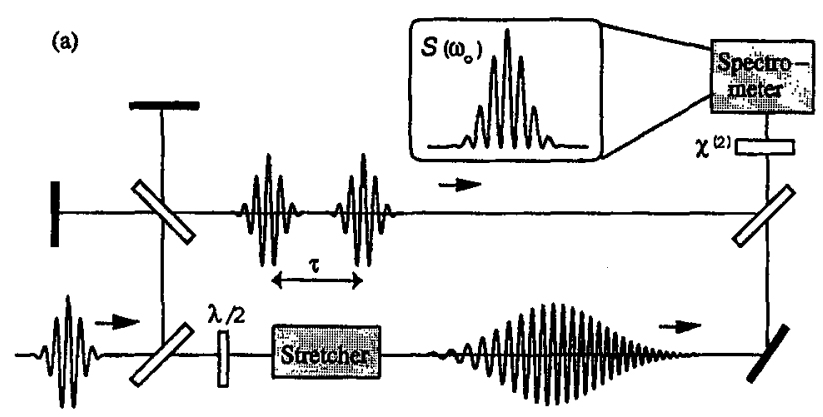

(b)

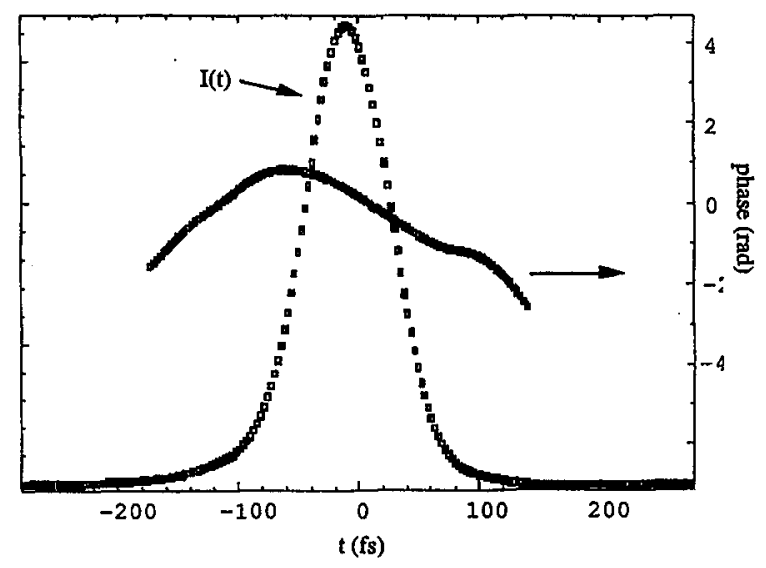

Fig. 6. (a) Sagnac interferometer for the measurement of the two-point correlation function of a time-stationary optical field. (See text for description.) (b) Magnitude of the experimentally measured correlation function for a filamentary broad-area laser diode.

A glass block mounted on a rotation stage is located in the common path allowing the Sagnac to be operated as a lateral shearing interferometer for both transverse coordinates. Also in the common-path region are first-order quarterand half-wave plates that are used for quadrature selection. The object fields are imaged onto a detector array by a lens, which must be placed after the interferometer so that phase structure due to the imaging optics does not appear in the measured interferograms ${ }^{\pi}$. The field exiting the input port of the beamsplitter is sent to a detector array. This arrangement ensures that both clockwise (cw) and counter-clockwise ( $\mathrm{ccw}$ ) fields experience one reflection from and one transmission through the beamsplitter, nulling the effect of deviations from $50 \%$ transmission and allowing for polarization insensitive orientation of the beamsplitter. The shear

IIf the shear is not introduced in the object space of the imaging system, then the interferograms exhibit tilt-like fringes perpendicular to the direction of shear that arise from the spherical phase front that is imposed on the field by the lenses. 
is varied from 0 to 1 (as a fraction of the full field) in equal steps. The resulting data consists of an array of numbers representing the measured intensity.

The two quadratures of $\Gamma\left(x, x^{\prime}\right)$ are determined by the following procedure. The quarter- and half-wave plates are oriented such that their fast axes are parallel to the object field polarization. In this orientation both $\mathrm{cw}$ and $\mathrm{ccw}$ fields travel equal optical path lengths. The intensity at the detector array is

$$
I_{\operatorname{det}}(y ; s)=\Gamma\left(y+\frac{s}{2}, y+\frac{s}{2}\right)+\Gamma\left(y-\frac{s}{2}, y-\frac{s}{2}\right)+2 \operatorname{Re} \Gamma\left(y+\frac{s}{2}, y-\frac{s}{2}\right),
$$

where $y$ is the position on the detector array, equal to the object field coordinate $x$ multiplied by the image magnification, and $s$ is the shear. Measurement of $I_{\operatorname{det}}(\mathrm{y} ; \mathrm{s})$ for all $s$ directly yields a sample of $\operatorname{Re} \Gamma\left(x, x^{\prime}\right)$ by subtracting out the individual intensities. Measurement of $\operatorname{Im} \Gamma\left(x, x^{\prime}\right)$ is accomplished by rotating the half-wave plate by 45 degrees so that the $\mathrm{cw}$ and $\mathrm{ccw}$ propagating fields experience different path lengths.

Measurement of two spatially separated Gaussian beams that were superposed with either fixed phases or random phases show that the two-point correlation function can be reconstructed quite reliably, and the integral degree of coherence $\mu$, defined in Eq. (4), evaluated. Note that $\mu$ provides a simple measure of beam quality, although a different one than the more usual $M^{2}$ parameter. It does not specify how close the beam is to a Gaussian, as does the latter value, but rather, whether it is possible in principle (although not of course necessarily in practice) to find an optical system that will produce a beam of size equal to the diffraction limited spot size of a Gaussian beam.

We have used this technique to examine filamentation in the output of broad-area semiconductor lasers; an important problem for the design of high power lasers of this type. Figure $6 \mathrm{~b}$ shows the correlation function for the output of an edge-emitting, AlGaAs, buried-heterostructure-type Fabry-Perot laser. The laser was operated about two times above threshold. The intensity at the output facet at this current was a four-peaked pattern, each peak centered at $x_{i}(i=1 \div 4)$, corresponding to four filaments being above threshold. The correlation function reveals, however, that these filaments are not all coherent with one another. Evidence for this is that the correlation function is close to zero in the regions near $\left(x_{3}, x_{i}\right)(i=1,2,4)$. The implication of this is that there exists no optical system that can transform this beam to a diffraction limited spot containing all of the laser's output power. Also, it shows that the filamentation of the laser beam is not necessarily due to a single mechanism. One might expect, for example, that if self-focusing-induced modulation instability were responsible for the filamentation then the degree of coherence of the filaments might depend only on $x_{i}-x_{j}$.

\subsection{Self-referencing interferometry for characterizing classical ultrashort optical pulses}

Interferometric characterization of ultrashort optical pulses has been performed primarily using the test-plus-reference geometry, in either the time domain [36], or the frequency domain, in which guise it is known as spectral interferometry [37-39]. 
Although some self-referencing methods have been developed for time-domain measurements, including the classic interferometric autocorrelation [40], and other methods [26] these either provide only partial phase information or require unreasonably precise temporal measurement. Precise spectral measurements are possible, though, and spectral shearing interferometry (SSI) [41, 42] is a self-referencing interferometric method that takes advantage of the direct phase retrieval routine offered by interferometry. It does require, however, shifting the two spectra with respect to one another by several hundred GHz. This can be done quite simply using upconversion.

The spectral interferogram of two pulses that are identical in all respects with the exception that they are shifted (sheared) in frequency with respect to one another, is given by the frequency domain analog of Eq. (12)

$$
\begin{aligned}
& S\left(\omega_{0}\right)=\left|\widetilde{E}\left(\omega_{0}\right)\right|^{2}+\left|\widetilde{E}\left(\omega_{0}+\delta \omega\right)\right|^{2}+2\left|\widetilde{E}\left(\omega_{0}\right) \widetilde{E}\left(\omega_{0}+\delta \omega\right)\right| \\
& \quad \times \cos \left(\phi_{\omega}\left(\omega_{0}+\delta \omega\right)-\phi_{\omega}\left(\omega_{0}\right)+\omega_{0} \tau\right),
\end{aligned}
$$

where $\tau$ is the temporal delay between the two replicas.
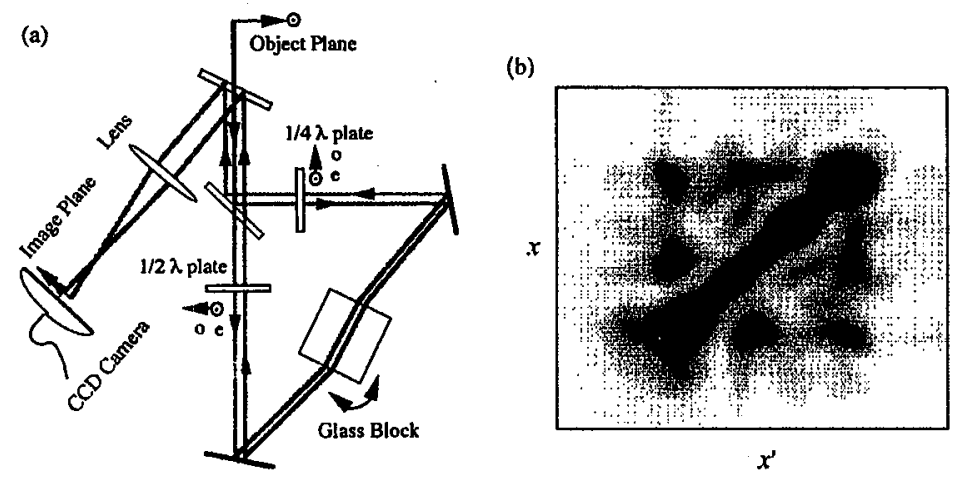

Fig. 7. (a) Upconversion spectral shearing interferometer for measuring the field of ultrashort optical pulses. (b) Phase and amplitude of an 80 fs-duration pulse from a modelocked laser measured using SSI.

Our apparatus for generating such a pair is shown in Fig. 7a. A portion of the pulse to be characterized is split off and directed through a Michelson interferometer. The output from the Michelson is a pair of test pulses separated in time by delay $\tau$. This pair of pulses is mixed with a stretched replica of the input pulse in a type-II nonlinear crystal $(250 \mu \mathrm{m} \mathrm{BBO})$. In the limit of large dispersion, the stretched pulse stretcher is highly chirped with each frequency occurring at a different time. Since the pulses in the test pair are delayed with respect to one another by $\tau$, each is upconverted with a different spectral slice of the stretched pulse. For a stretched Gaussian pulse of temporal duration $T$ and spectral width $\Omega$, the spectral shear between the upconverted pair of test pulses is $\delta \omega=\tau \Omega / T$. It is necessary that $T \gg \tau$ so that the frequency of the chirped pulse does not change over the duration of an individual unstretched test pulse. The resultant spectral interferogram is recorded by a spectrometer. 
The phase reconstruction routine follows a procedure introduced by Takeda et al. [43]. The recorded spectral interferogram is Fourier transformed with respect to $\omega$, and all features at times near to and less than $t=0$ are discarded. The remaining signal for positive $t$ is inverse transformed. After subtracting the carrier frequency term $\omega_{0} \tau$ from the resulting phase distribution we are left with the relative phase, $\phi_{\omega}\left(\omega_{0}+\delta \omega\right)-\phi_{\omega}\left(\omega_{0}\right)$, between each pair of frequency components separated by $\delta \omega$. (In the limit of small $\delta \omega$ this is approximately the group delay at $\omega_{0}$.)

Using the above apparatus and inversion procedure we reconstructed the amplitude and phase of the pulses output from a Tissapphire oscillator. The test pulses leaving the Michelson arrangement are separated by roughly $4 \mathrm{ps}$. The dispersed pulse is stretched to $25 \mathrm{ps}$, roughly a factor of 300 , such that a spectral shear of $16 \%$ of the total pulse bandwidth is achieved and upconversion of the test pulses with "continuous-wave slices" of the stretched pulse is assured. The reconstructed pulse amplitude and phase in the time domain is shown in Fig. 7b.

\subsection{Self-referencing interferometry for characterizing atomic wave functions}

Shearing interferometry can also be used to measure the transverse component of the wave function of the center of mass degree of freedom of an atom moving in free space. Freyberger et al. [44] have recently proposed a method quite similar to that described for optical fields in Sec. 4.1. Here we discuss an alternate approach that makes use of shearing in momentum space rather than in configuration space [45].

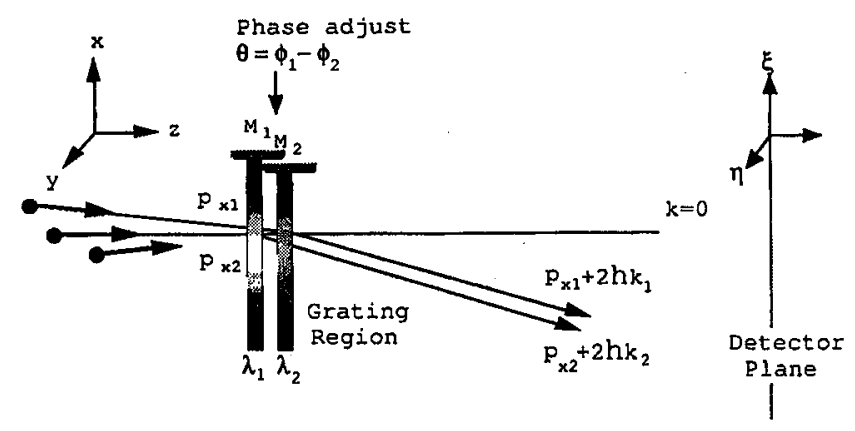

Fig. 8. Double-grating lateral shearing interferometer for measuring the density matrix of the transverse coordinate of free atoms. The $\lambda_{i}$ 's are the wavelengths of the two standing-wave light field diffraction gratings, whose relative phases are adjusted using the mirrors $M_{i}$. Monoenergetic atoms with different transverse momenta area scattered into the same direction $\left(p_{x i}+2 \hbar k_{i}\right)$ and detected in the far-field at $\xi$.

The set up is sketched in Fig. 8. It consists of a beam of atoms, width $\Delta x$ in their ground electronic states, with reasonable collimation, propagating in the $z$-direction. It is incident upon two quasi-monochromatic standing wave light fields with wave vectors in the $\pm x$ directions. The fields differ in their wavelength, and one has a phase adjustment that allows the nodes of the standing wave to be 
shifted through one half wavelength. An array of hot-wire or other atom detectors is situated in the far-field of the apparatus.

If the standing light waves are strongly detuned from the atomic resonance, and the gratings, width $\epsilon_{i}$, are taken to be thin, and if the two gratings are closely spaced, so that there is no lateral spreading of the beam within the grating region due to scattering or diffraction, then the wave function of the beam immediately to the right of the second grating is

$$
\Psi\left(x, z=\epsilon_{1}+\epsilon_{2}\right)=\Psi_{+}(x, 0)=T_{1}(x) T_{2}(x) \Psi(x, 0),
$$

where $T_{i}(x)$ is the grating (spatial) response function. Of course, the atom also accumulates a phase $\Phi_{i}=E \tau_{i} / \hbar$, where $\tau_{i}$ is the approximate time taken for the atom to traverse the grating.

If the beam is then allowed to propagate in free space then the wave function $\Psi(\xi, z)$ at the detector plane, in the far-field of the gratings, is proportional to the Fourier transform of the wave function immediately after the gratings. The detected signal in the vicinity of the first-order scattered beam is approximately

$$
\begin{aligned}
& S_{1}(\xi)=\left|A_{10}\right|^{2}\left\{\tilde{\widetilde{\rho}}\left(\frac{k \xi}{z}-2 k_{1}, \frac{k \xi}{z}-2 k_{1}\right)^{2}+\tilde{\widetilde{\rho}}\left(\frac{k \xi}{z}-2 k_{2}, \frac{k \xi}{z}-2 k_{2}\right)^{2}\right. \\
& \left.\quad+2 \operatorname{Re}\left[\mathrm{e}^{\left(\phi_{2}-\phi_{1}\right)} \tilde{\tilde{\rho}}\left(\frac{k \xi}{z}-2 k_{1}, \frac{k \xi}{z}-2 k_{2}\right)\right]\right\} .
\end{aligned}
$$

This part of the signal depends on the momentum space correlation function of the initial state, and from it the momentum representation of the initial density matrix can be reconstructed, even for mixed states. The requirement of well-separated first-order diffraction sets a lower bound on the wave functions that can plausibly be reconstructed using this technique. In particular the scattering light beams ought to satisfy $k_{1}, k_{2}>2 \pi / \Delta x$.

A procedure for the complete reconstruction of this quantity follows from Eq. (15), and is quite analogous to that described in Sec. 4.2 for the optical field. The two quadratures of the density matrix are measured by adjusting the phase between the two gratings to be first 0 then $\pi / 2$. Simple estimates indicate that this method should be experimentally feasible for sodium atoms moving at about $10 \mathrm{~m} / \mathrm{s}$, and having a divergence of less than $2.5 \mathrm{mrads}$, using two laser beams tuned near the $D$ lines, that may be detuned from one another by about $50 \mathrm{~nm}$ while maintaining a relatively constant Rabi frequency.

\section{Interferometric spectrography}

\subsection{Test-plus-reference interferometry for quantum state reconstruction of ultrashort optical pulses}

As an example of the importance of both classical and quantum field measurements we discuss in this section a method for measuring the multimode quantum state of a pulsed quantum optical field. The single mode problem has been solved using optical homodyne tomography, (OHT) [4] and partial reconstruction has been developed for the two-mode problem [46]. As Raymer has shown [48], a 
complete state reconstruction for even the two-mode problem using OHT is unpleasantly cumbersome. However, it is possible to reconstruct the $Q$-function of a multimode field using a simple multi-detector apparatus, and using a much simpler arrangement [48].

Consider a balanced test-plus-reference spectral interferometer, into which the quantum (test) pulse and a classical (reference or local oscillator (LO)) pulse, duration $\tau_{\mathrm{p}}$, enter. The spectra of the radiation at the two output ports of the beamsplitter are detected by two multichannel detector arrays, and the difference photocount numbers $N_{\omega_{i}}$ are taken channel by channel from the array outputs. The discrete Fourier transform of the $N_{\omega_{i}}$ is taken to yield a set of complex numbers $N_{t_{i}}$ [49]. The real and imaginary parts of this set of numbers are realizations of the two quadratures of a set of temporal modes of the field, each displaced by a time $\mathrm{i} \tau_{\mathrm{p}}$ from the reference pulse. This procedure is followed on each shot, and the statistics of the set $N_{t_{i}}$ constructed. Then it can be shown that the probability distribution of these numbers scaled by the LO field amplitude is proportional to the joint $Q$-function for the set of temporal modes

$$
P\left(\left[N_{t_{i}}\right]\right)=Q\left(\left[X_{t_{i}}=\operatorname{Re} \frac{N_{t_{i}}}{2 E} ; Y_{t_{i}}=\operatorname{Im} \frac{N_{t_{i}}}{2 E}\right]\right),
$$

where $X_{t_{i}}$ and $Y_{t_{i}}$ are the in-phase and in-quadrature field amplitudes for mode $t_{i}$. Note that the single or two mode statistics can be obtained by simply tracing over the remaining modes. The total number of modes on which information is simultaneously obtained is equal to the number of pixels (or resolution elements) of the multichannel detectors.

Note that the spectral interferogram is sensitive only to the modulus of the delay of the LO pulse from the test pulse. Thus there is an uncertainty as to whether the detected photons came from a temporal mode that arrived at the detector before the LO pulse or after it. This is analogous to the case in heterodyne detection in which the measurement space is increased to include the image modes of the test field. Shapiro and Wagner [50] have shown that this is equivalent to making a joint measurement of the two quadratures of the signal mode simultaneously provided the image mode is in the vacuum, so that measurement of the multimode Wigner function of the test field is not possible.

\section{Conclusions}

The similarity of detectors and dynamic equations for both quantum matter waves and classical optical fields allows techniques developed for the characterization of one to be easily translated to the characterization of the other. We have illustrated this by several experimental and numerical examples. Note that both characterization procedures are necessary when measuring the state of a quantum field, since then one must first characterize the classical mode function in which the quantum system exists. The important and emerging problem of quantum state engineering in both matter and radiation will no doubt benefit from the ability to measure fields of all types, although new methods need to be developed to tackle the multimode problem in matter. Given the recent activity and progress in this field, it is likely that such techniques will be available in the near future.

This work was supported by the National Science Foundation. 


\section{References}

[1] J.H. Eberly, C.K. Law, Acta Phys. Pol. A 93, (1998).

[2] V.B. Braginsky, F.Ya. Khalili, Quantum Measurement, Cambridge University Press, Cambridge (NY) 1992.

[3] U. Leonhardt, M.G. Raymer, Phys. Rev. Lett. 76, 1989 (1996).

[4] D.T. Smithey, M. Beck, J. Cooper, M.G. Raymer, Phys. Scr. T 48, 35 (1993).

[5] K. Wodkiewicz, private communication.

[6] I.A. Walmsley, V. Wong, J. Opt. Soc. Am. B 13, 2453 (1996).

[7] M.G. Raymer, Am. J. Phys. 62, 10 (1994).

[8] W. Vogel, D.-G. Welsch, Lectures on Quantum Optics, Akademie Verlag, Berlin/VCH Publishing Inc., New York 1994.

[9] U. Leonhardt, Measuring the Quantum State of Light, Cambridge University Press, Cambridge 1997.

[10] S. Stenholm, Ann. Phys. (New York) 218, 233 (1980).

[11] A. V. Vinogradov, J. Janszky, Phys. Rev. Lett. 64, 2771 (1990).

[12] J. Janszky, A.V. Vinogradov, I.A. Walmsley, J. Mostowski, Phys. Rev. A 50, 1777 (1994).

[13] I.A. Walmsley, M.G. Raymer, Phys. Rev. A 52, 681 (1995).

[14] T.J. Dunn, I.A. Walmsley, S. Mukamel, Phys. Rev. Lett. 74, 884 (1995).

[15] J.H. Eberly, K. Wodkiewicz, J. Opt. Soc. Am. 67, 2591 (1979).

[16] P. Kowalczyk, C. Radzewicz, J. Mostowski, I.A. Walmsley, Phys. Rev. A 42, 5622 (1990).

[17] A. Assion, M. Geisler, J. Helbing, V. Seyfried, T. Baumert, Phys. Rev. A 54, R4605 (1996).

[18] L. Waxer, I.A. Walmsley, W. Vogel, Phys. Rev. A 56, R3491 (1997).

[19] I. Walmsley, R. Trebino, Opt. Photonics News 7, 23 (1996).

[20] D.J. Kane, R. Trebino, IEEE J. Quantum Electron. 29, 571 (1993).

[21] J.-P. Foing, J.-P. Likforman, M. Joffre, A. Migus, IEEE J. Quantum Electron. 28, 2285 (1992).

[22] S.P. LeBlanc, R. Sauerbrey, Opt. Commun. 111, 297 (1994).

[23] J.-K. Rhee, T.S. Sosnowski, A.-C. Tien, T.B. Norris, in: Conf. Lasers and Electro-Optics, Baltimore (MD), 1995, OSA, Washington (DC) 1995, paper CPD39.

[24] V. Wong, I.A. Walmsley, J. Opt. Soc. Am. B 14, 944 (1997).

[25] J.L.A. Chilla, O.E. Martinez, IEEE J. Quantum Electron. 27, 1228 (1991).

[26] K.C. Chu, J.P. Heritage, R.S. Grant, K.X. Liu, A. Dienes, W.E. White, A. Sullivan, Opt. Lett. 20, 904 (1995).

[27] K.W. DeLong, R. Trebino, J. Hunter, W.E. White, J. Opt. Soc, Am. B 11, 2206 (1994).

[28] K.W. DeLong, D.N. Fittinghoff, R. Trebino, B. Kohler, K. Wilson, Opt. Lett. 19, 2152 (1994).

[29] D. Kohler, L. Mandel, J. Opt. Soc. Am. 63, 126 (1972).

[30] P. DeSantis, F. Gori, G. Guattari, C. Palma, J.M. Webster, J. Photo. Sc. 33, 197 (1985). 
[31] N. Streibl, Opt. Commun. 49, 6 (1984).

[32] K. Ichikawa, A.W. Lohmann, M. Takeda, Appl. Opt. 27, 3433 (1988).

[33] K.H. Brenner, A.W. Lohmann, Opt. Commun. 42, 310 (1982).

[34] K. Creath, in: Progress in Optics XXVI, Ed. E. Wolf, Elsevier, Amsterdam 1988, p. 349 .

[35] C. Iaconis, I.A. Walmsley, Opt. Lett. 21, 1783 (1996).

[36] J.E. Rothenberg, D. Grischkowsky, Opt. Lett. 12, 99 (1987).

[37] E. Fung, E.S. Yeung, Analytical Chem. 67, 1913 (1995).

[38] L. Lepetit, G. Cheriaux, M. Joffre, J. Opt. Soc. Am B 12, 2467 (1995).

[39] D.N. Fittinghof, J.L. Bowie, J.N. Sweetser, R.J. Jennings, M.A. Krumbügel, K.W. Delong, R. Trebino, I.A. Walmsley, Opt. Lett. 21, 884 (1996).

[40] J.C. Diels, E.W. VanStryland, D. Gold, in: Proc. First Intern. Conf. on Picosecond Phenomena, Eds. C.V.Shank, E.P. Ippen, S.L. Shapiro, Springer, New York 1978, p. 117.

[41] V.A. Zubov, T.I. Kuznetsova, Laser Physics 2, 73 (1992).

[42] V. Wong, I.A. Walmsley, Opt. Lett. 19, 287 (1994).

[43] M. Takeda, H. Ina, S. Kobayashi, J. Opt. Soc. Am. 72, 156 (1982).

[44] M. Freyberger, S. Kienle, V. Yakovlev, Phys. Rev. A 56, 195 (1997).

[45] I.A. Walmsley, N.P. Bigelow, submitted for publication.

[46] D.F. McAlister, M.G. Raymer, Phys. Rev. A 55, R1609 (1996).

[47] M.G. Raymer, M. Beck, D.F. McAlister, Phys. Rev. Lett. 72, 1137 (1994).

[48] I.A. Walmsley, C. Iaconis, R. Trebino, in: Ultrafast Phenomena X, Eds. P. Barbara, J. Fujimoto, Springer, New York 1996, p. 169.

[49] M.G. Raymer, J. Cooper, M. Beck, Phys. Rev. A 48, 4617 (1993).

[50] J.H. Shapiro, S.S. Wagner, IEEE J. Quantum Electron. QE-20, 803 (1984). 